\title{
The validity of the INR system for patients with liver disease
}

\author{
Armando Tripodi
}

Published online: 17 September 2010

(C) Springer Science+Business Media, LLC 2010

I read with interest the paper by Wei et al. [1], published recently in the Journal of Thrombosis and Thrombolysis on the assessment of the validity of the INR system for patients with liver disease and wish to make comments. The authors measured the INR with different commercial thromboplastins for a cohort of patients with liver disease and a cohort of patients stabilized on vitamin $\mathrm{K}$ antagonists (VKA). While the between-thromboplastin INRs for the patients with liver disease were significantly different, those for the patients on VKA were not. The obvious conclusion is that the INR (as it is calibrated for patients on VKA and here called INRvka) is not valid for patients with liver disease and, therefore, the model of end stage liver disease (MELD) score, once proposed as an objective index to prioritize patients for liver transplantation, would not allow parity of organ allocation. In their discussion Wei et al. [1], ignored at least seven of the many papers published over the last few years on this topic. Here few examples. Trotter et al. [2], were among the first in 2004 to show that the MELD score was not effective in securing parity of organ allocation. Other papers have consistently shown (from 1994 to 1999) that the INR, devised for patients on VKA, is not valid for patients with chronic liver disease [3, 4]. In 2007, Tripodi et al. [5] and Bellest et al. [6] reported independently on the same issue of Hepatology that the INRvka is not valid for patients with chronic liver disease as shown by the fact that the MELD, calculated by including in the equation the INRvka, depends on

\footnotetext{
A. Tripodi $(\bowtie)$

Department of Internal Medicine, Angelo Bianchi Bonomi Hemophilia and Thrombosis Center, IRCCS Cà Granda Ospedale Maggiore Policlinico Foundation and Università degli Studi di Milano, Milan, Italy

e-mail: armando.tripodi@unimi.it
}

the thromboplastin used for testing. In the same papers these authors have independently shown that an alternative system of ISI calibration, provisionally called ISIliver (as opposed to the ISIvka) [5] can be obtained by inserting into the calibration plot, plasmas from patients with chronic liver disease instead of plasmas from patients on VKA [5, 6]. This alternative system of calibration proved effective in minimizing between-thromboplastin MELD results. More recently, Sermon et al. [7], confirmed these results, and Tripodi et al. [8] extended this model of ISIliver calibration to portable coagulation monitors. Finally, a review article on this topic has been published in the Journal of Thrombosis and Haemostasis in 2009 [9] and official recommendations have been issued independently by the International Society on Thrombosis and Haemostasis [10] and by the American Journal of Transplantation [11]. Surprisingly, some of the above papers $[2-6,8,9]$ that appeared in the literature well before the publication of Wei et al. [1] have escaped their attention.

\section{References}

1. Wei Y, Li J, Zhang L, Zheng D, Shi B, Cong Y (2010) Assessment of validity of INR system for patients with liver disease associated with viral hepatitis. J Thromb Thrombolysis 30:84-89

2. Trotter JF, Brimhall B, Arjal R (2004) Specific laboratory methodologies achieve higher model for end stage liver disease (MELD) scores for patients listed for liver transplantation. Liver Transplant 10:995-1000

3. Kovacs MJ, Wong A, MacKinnon K, Weir K, Keeney M, Boyle E, Cruickshank M (1994) Assessment of the validity of the INR system for patients with liver impairment. Thromb Hemost 71:727-730

4. Denson KW, Reed SV, Haddon ME, Woodhams B, Brucato C, Ruiz J (1999) Comparative studies of rabbit and human recombinant tissue factor reagents. Thromb Res 94:255-261 
5. Tripodi A, Chantarangkul V, Primignani M, Fabris F, Dell'Era A, Sei C, Mannucci PM (2007) The international normalized ratio calibrated for cirrhosis $\left(\mathrm{INR}_{\text {liver }}\right)$ normalizes prothrombin time results for model for end-stage liver disease calculation. Hepatology 46:520-527

6. Bellest L, Eschwege V, Poupon R, Chazouillères O, Robert A (2007) A modified international normalized ratio as an effective way of prothrombin time standardization in hepatology. Hepatology 46:528-534

7. Sermon AM, Smith JM, Maclean R, Kitchen S (2010) INR in liver disease: an ISI derived from liver disease patients improves agreement between INRs with different reagents. Thromb Haemost 103:757-765

8. Tripodi A, Chantarangkul V, Primignani M, Dell'Era A, Clerici M, Iannuzzi F, Aghemo A, Cazzaniga M, Salerno F, Mannucci PM (2009) Point-of-care coagulation monitors calibrated for the international normalized ratio for cirrhosis $\left(\mathrm{INR}_{\text {liver }}\right)$ can help to implement the $\mathrm{INR}_{\text {liver }}$ for the calculation of the MELD score. J Hepatol 51:288-295

9. Tripodi A, Chantarangkul V, Mannucci PM (2008) The international normalized ratio to prioritize patients for liver transplantation. Problems and possible solutions. J Thromb Haemost 6:243-248

10. Tripodi A, Baglin T, Robert A, Kitchen S, Lisman T, Trotter JF, on behalf of the Subcommittee on Control of Anticoagulation of the Scientific and Standardisation Committee of the International Society on Thrombosis and Haemostasis (2010) Reporting prothrombin time results as international normalised ratios for patients with chronic liver disease. J Thromb Haemost 8:1410-1412

11. Porte RG, Lisman T, Tripodi A, Caldwell SH, Trotter JF, and the Coagulation in Liver Disease Study Group (2010) The international normalized ratio (INR) in the MELD score: problems and solutions. Am J Transplant 10:1349-1353 